\title{
Rabaska
}

Revue d'ethnologie de l'Amérique française

\section{Ancelet, Barry-Jean et Kristi Guillory. Louisiana Folk Masters: \\ Women's Home Music. Lafayette, Louisiane, Louisiana \\ Crossroads Records, 2007. 2 DC +28 p.}

\section{Ronald Labelle}

Volume 6, 2008

URI : https://id.erudit.org/iderudit/019997ar

DOI : https://doi.org/10.7202/019997ar

Aller au sommaire du numéro

Éditeur(s)

Société québécoise d'ethnologie

ISSN

1703-7433 (imprimé)

1916-7350 (numérique)

Découvrir la revue

Citer ce compte rendu

Labelle, R. (2008). Compte rendu de [Ancelet, Barry-Jean et Kristi Guillory.

Louisiana Folk Masters: Women's Home Music. Lafayette, Louisiane, Louisiana

Crossroads Records, 2007. 2 DC + 28 p.] Rabaska, 6, 151-153.

https://doi.org/10.7202/019997ar d'utilisation que vous pouvez consulter en ligne.

https://apropos.erudit.org/fr/usagers/politique-dutilisation/ 


\section{Comptes rendus}

Ancelet, Barry-Jean et Kristi Guillory. Louisiana Folk Masters : Women's Home Music. Lafayette, Louisiane, Louisiana Crossroads Records, 2007. $2 \mathrm{DC}+28 \mathrm{p}$.

La parution d'un coffret présentant sur disques des enregistrements sonores provenant d'archives est un événement rare. De telles productions s'adressent généralement à un public limité, car ce n'est pas monsieur tout le monde qui peut apprécier des enregistrements de terrain présentant des chants traditionnels interprétés sans arrangement musical. Voilà une première raison pour se réjouir à la parution de ce beau coffret présentant 41 chansons enregistrées en Louisiane entre 1937 et 1995.

Il est facile d'oublier que la Louisiane a hérité d'un riche bagage de chansons traditionnelles françaises, tellement nous avons été saturés de musique «cajun » contemporaine où l'on nous exhorte à « laisser le bon temps rouler ». Barry-Jean Ancelet explique dans l'introduction que la musique cadienne contemporaine servant essentiellement à accompagner la danse, les chansons interprétées dans un contexte intime, ce qu'il appelle home music, ont été quelque peu délaissées.

L'échantillon présenté ici témoigne d'une grande diversité de chansons, allant des anciennes complaintes comme La Vaillante Catherine (une version de Les Anneaux de Marianson) jusqu'aux chansons légères comme Quel petit homme, une variante du Petit Mari. On y trouve quelques chansons comme Mon bon vieux mari et L'Oiseau dans le nid qui ont été popularisées par des chanteurs bien connus au $\mathrm{Xx}^{\mathrm{e}}$ siècle, mais il y a beaucoup de surprises aussi, comme la chanson populaire française La Madelon, qui date de l'époque de la Première Guerre mondiale.

L'aspect le plus original de Women's Home Music est le fait que toutes les chansons proviennent de femmes, alors que nous associons généralement la musique cadienne à des chanteurs masculins comme Nathan Abshire, les frères Balfa et Clifton Chénier. Barry Ancelet et Kristi Guillory expliquent que l'idée leur est venue suite à la réalisation récente de deux thèses de maitrise consacrées à des chanteuses traditionnelles cadiennes. Au total, onze chanteuses sont représentées sur les disques. La majorité des pièces proviennent soit des enregistrements de terrain effectués par Barry Ancelet 
pendant les années 1970, ou de la collection Harry Oster qui date des années 1950. On y trouve neuf chansons par Alma Barthélémy, qui s'est fait connaître lors de sa participation au Festival of American Folklife à Washington pendant les années 1970, mais il y a aussi plusieurs chanteuses qui n'ont jamais interprété leur répertoire en dehors du foyer. C'est le cas de la chanteuse créole Inez Catalon qui a été enregistrée par Barry Ancelet dans sa cuisine.

Étant donné qu'Ancelet a personnellement connu la plupart des chanteuses, il a pu recueillir d'elles de l'information précieuse sur le contexte de transmission de la chanson traditionnelle. Odile Falcon, par exemple, lui a raconté que sa famille se rassemblait sur la galerie chaque soir et que chacun chantait à son tour : " C'est avec mon grand-père et ma grand-mère que j'ai appris ça. On s'assisait tous les soirs jusqu'à onze heures du soir. Ça chantait tout[es] les chansons pour nous autres ». Lula Landry, dont dix chansons sont reproduites ici, racontait à Barry Ancelet comment il lui suffisait d'entendre une chanson une seule fois pour l'apprendre en se la répétant dans sa tête pour la graver dans sa mémoire.

Les disques compacts comprennent, en plus des chansons, quelques brefs extraits d'entrevues où les interprètes racontent leurs souvenirs. Des transcriptions complètes des chansons, ainsi que les entrevues, sont présentées dans un texte de 42 pages disponible dans un fichier électronique en format pdf. Cet ajout solutionne le problème de manque d'espace dans le petit cahier de 28 pages qui accompagne les disques et où Barry Ancelet et ses collaborateurs introduisent les chanteuses et leur répertoire, en plus de situer chaque chanson au point de vue historique à l'aide de références à des travaux comme ceux de Conrad Laforte.

Le format standard du disque compact commercial impose malheureusement des contraintes dans un projet qui vise à ajouter de la documentation écrite à des disques sonores. Une solution originale a été adoptée ici, soit l'ajout d'un fichier pdf. Ce fichier sera très apprécié par les personnes voulant apprendre les chansons. On a même pris la peine de traduire entièrement en anglais les paroles des chansons et les extraits d'entrevues pour que les auditeurs unilingues anglophones puissent mieux les comprendre.

Le tout constitue une production très soignée qui est évidemment le fruit d'un long travail de préparation et de réflexion. Le résultat constitue une autre preuve de l'importance de trouver des moyens pour valoriser les archives de folklore en rendant leurs collections disponibles. Dans la foulée des nouvelles technologies, il n'est pas facile de décider quels formats conviennent le mieux à de tels projets, mais la toile, le disque compact, le DvD, le fichier sonore mp3 et le fichier texte pdf offrent tous de nouveaux outils pouvant servir à diffuser le savoir traditionnel. Louisiana Folk Masters : Women's 
Home Music est une belle réalisation qui nous présente un exemple d'un modèle pratique pouvant inspirer d'autres projets du genre.

Ronald Labelle

Université de Moncton

Arsenault, Georges. La Mi-Carême en Acadie. Tracadie-Sheila, La Grande Marée, 2007, 160 p. ISBN 978-2-349-72254-6.

Depuis plusieurs années Georges Arsenault étudie les traditions acadiennes de son milieu, l'Île-du-Prince-Édouard. Il s'intéresse surtout à faire revivre les coutumes disparues ou presque oubliées, ou à les dévoiler à ceux qui n'ont pas eu la chance de les vivre. Après la publication de son livre Courir la chandeleur en 1982, il aspirait à produire une série de petits livres sur les diverses coutumes de ce peuple. Finalement, en 2005, il lançait ce projet en publiant un premier recueil, Noël en Acadie, aux éditions La Grande Marée, et ce fut un grand succès. Après avoir capté l'intérêt du public en lui présentant une fête populaire toujours vivante et célébrée un peu partout dans le monde, il choisit pour son deuxième ouvrage un sujet moins connu qui le passionne depuis longtemps : la mi-carême. La Mi-Carême en Acadie montre l'évolution d'une ancienne tradition française qui a survécu dans quelques communautés acadiennes et québécoises. En fait, la mascarade hivernale a connu un essor considérable dans quelques régions telles que Chéticamp et Saint-Joseph-du-Moine, au Cap-Breton, et Natashquan, au Québec, où la fête s'est étendue sur une semaine entière et prend la forme d'un véritable festival. Ce recueil ethnographique, qui propose un regard minutieux sur tous les aspects de la tradition acadienne, partage des souvenirs intimes et encore bien vifs de la fête ; pour plusieurs, la mi-carême représente la nostalgie de la vie d'antan, mais pour d'autres c'est une réalité vivante. $L a$ Mi-Carême en Acadie est un ouvrage de référence dont le but principal est de renouveler les connaissances sur une tradition presque complètement disparue dans la majorité des régions acadiennes en espérant la faire revivre encore. L'auteur, qui a vécu la tradition chez lui à l'Île-du-Prince-Édouard, a déjà essayé de ravigoter la tradition dans son milieu en publiant des articles sur le sujet et même en courant avec quelques amis, mais sans véritable succès. Comme il le souligne dans son avant-propos, cette publication représente une autre tentative de redonner vie à la tradition ancienne : "Incontestablement, ce livre s'avère la suite logique de mes démarches pour conserver la mémoire de cette vénérable tradition, de la faire mieux connaître et même de la faire revivre » (p. 14). 